\title{
7 Veränderung von Berufen im Gesundheitswesen durch E-Health
}

\author{
Klaus Juffernbruch
}

Das digitale Gesundheitswesen ist patientenzentriert.

\section{Bestandsaufnahme und Stellenwert}

Die rasante Entwicklung digitaler Technologien hat in vielen Branchen nicht nur eine evolutionäre Weiterentwicklung bestehender Geschäftsmodelle gebracht, sondern in vielen Fällen zu radikal neuen, disruptiven Geschäftsprozessen geführt, die die alten Modelle ergänzen oder sogar verdrängen.

So hat beispielsweise Amazon erst den Buchhandel und später den gesamten Einzelhandel revolutioniert, Uber fordert das Taxigewerbe heraus so wie Airbnb die Hotelbranche.

Gleichzeitig beschert die immer leistungsfähigere und preiswertere Digitaltechnik jedem Einzelnen Möglichkeiten, die vorher nur Profis vorbehalten waren. OnlinePlattformen verkaufen nicht nur Literatur, sondern ermöglichen auch jedem, als Autor eigene Bücher weltweit zu publizieren. Digitale Kameras, Laptop und Videoplattformen wie YouTube und Vimeo machen jeden zum Regisseur, Produzenten und Betreiber einer Art Fernsehsender. Auf ebay und Amazon kann jeder zum Händler werden. Ähnliche, tiefgreifende Umwälzungen durch die Digitalisierung haben auch im Cesundheitswesen begonnen und werden immer mehr Berufsgruppen erfassen und verändern. 
Zentrale Bedeutung kommt dabei diesen fünf Faktoren zu:

- Kommunikationstechnologien

- Big Data und künstliche Intelligenz

- Miniaturisierung von Sensoren

- Robotik

- 3D-Druck

\section{Kommunikationstechnologien}

Zu den Kommunikationstechnologien allgemein zählen alle Formen der Textübertragung, wie beispielsweise E-Mail, SMS und WhatsApp, Audioübertragung, wie Telefonate, WhatsApp-Sprachnachrichten oder Skype-Telefonate, Videokommunikation, wie z.B. FaceTime, Skype, Telepresence, sowie gemischte Formen der Kommunikation, bei denen mehrere Kanäle gleichzeitig genutzt werden, zu denen beispielsweise Webkonferenzsysteme zählen, die Audio-, Video-, Text- und Datenübertragung vereinen.

Immer leistungsfähigere Endgeräte in Form von PCs und Smartphones verbunden mit immer schnelleren drahtlosen und kabelgebundenen Netzwerken ermöglichen es jedem Patienten und jeder Berufsgruppe im Gesundheitswesen, von den Vorteilen dieser modernen Kommunikationsformen zu profitieren.

Beim Telemonitoring nutzt man Netzwerke, um Daten des Patienten von medizinischen Messgeräten zum Therapeuten zu übertragen. Das können Blutdruckwerte, Gewicht, Herzfrequenz, Elektrokardiogramm (EKG) u. Ä. sein. Die Entfernung zwischen Behandler und Patient spiel dabei keine Rolle. Auch kann die Datenübertragung von zu Hause, der Arbeitsstelle und sogar von unterwegs erfolgen.

Bei Telekonsultationen sind medizinische Berufsgruppen per Audio- und Videoübertragung untereinander beziehungsweise mit den Patienten verbunden. Das ermöglicht schnelle und ortsunabhängige Besprechungen auch mit Spezialisten, die nicht in jedem Ort oder jedem Krankenhaus verfügbar sind.

Viele europäische und außereuropäische Länder nutzen bereits seit etlichen Jahren vollumfängliche Online-Konsultationen zwischen Patienten und Ärzten. Führend sind hier u.a. die skandinavischen Länder, Großbritannien und die Schweiz.

In Deutschland ist aufgrund der restriktiven Bestimmungen in der Musterberufsordnung der Ärzte sowie der 4. Novellierung des Arzneimittelgesetzes (AMG-Novelle) Fernbehandlung nur sehr eingeschränkt möglich.

\section{Big Data und künstliche Intelligenz}

Schnellere Prozessoren und größere Massenspeicher erlauben die Erfassung und Auswertung von immer größeren Datenmengen. In der Medizin führt diese Technologie zum Aufdecken neuer Zusammenhänge, z.B. zwischen Genomdaten und klinischen Daten. Durch diese Erkenntnisse können Diagnostik und Therapie zunehmend individueller auf den einzelnen Patienten zugeschnitten werden. Diese personalisierte Medizin bringt eine gezieltere Behandlung bei reduzierten Nebenwirkungen. Big Data ist außerdem die Grundlage für Systeme der künstlichen Intelligenz (KI). 
Methoden der künstlichen Intelligenz ermöglichen es Computern, natürliche Sprache in gesprochener und schriftlicher Form zu verstehen. Dadurch können sie unstrukturierte Daten, z.B. in Zeitschriftenartikeln, auswerten und in strukturierte Formen überführen und Schlussfolgerungen daraus ziehen. Intelligente Mustererkennung erlaubt die automatische Identifikation von Gegenständen und Personen. In der Medizin können sie pathologische Merkmale auf Röntgenbildern finden und große Datenmengen auf verborgene Zusammenhänge hin untersuchen. Damit können sie Ärzten bei Diagnosefindung und Therapieplanung Entscheidungsunterstützung geben.

\section{Miniaturisierung von Sensoren}

Zunehmend kleinere und erschwinglichere Sensoren erlauben es jedem Gesundheitsinteressierten, daheim und unterwegs eine Vielzahl medizinischer Daten selbst zu erheben, für die früher ein Besuch beim Arzt oder im Krankenhaus notwendig war. Smartwatches und Smartphones entwickeln sich damit zu einer Art „Schweizer Taschenmesser" des Gesundheitswesens. Mit entsprechenden Zusätzen wird ein Smartphone zu Pulsmesser, EGK, Mikroskop, Ophthalmoskop, Fetalem Doppler, Blutdruckund Blutzuckermessgerät und vielem mehr.

\section{Robotik}

Kleinere Mechanik mit Sensoren, künstlicher Intelligenz und leistungsfähigen Akkus bringt uns immer neue nützliche Alltagshelfer vom Staubsaugroboter bis zum selbstfahrenden Auto. In der Medizin helfen Roboter bei Operationen und entlasten das Personal in Pflege und Reha von körperlich schweren Tätigkeiten.

\section{D-Druck}

Laser- und Tintenstrahldrucker haben die Erstellung hochwertiger und individueller Druckerzeugnisse für viele Zwecke aus der Druckerei in die Wohnung oder das Büro verlagert.

Eine ähnliche Entwicklung bahnt sich im Bereich der Fertigung an. Mit 3D-Drucktechnik werden maßgeschneiderte Implantate und Prothesen für Patienten hergestellt. Sogar mit 3D-Druck von lebenden Geweben wurden erste Erfolge erzielt.

\section{Beschreibung des Transformationsprozesses}

Die beschriebenen IT-gestützten Technologien haben das Potenzial, praktisch alle Berufe im Cesundheitswesen tiefgreifend und nachhaltig zu verändern, wie im Folgenden an Hand ausgewählter Beispiele erläutert wird.

\section{Ärztliche und therapeutische Berufe}

Durch Kommunikationstechnologien können Therapeuten und Patienten unabhängig von Zeit und Ort in Austausch treten. Für Berufe wie Ärzte, Psychotherapeuten, 
Logopäden und teilweise auch Physiotherapeuten ermöglicht das neue Modelle der Berufsausübung von zu Hause aus, einschließlich flexiblerer Teilzeitgestaltungen. So können mehr qualifizierte Menschen mit familienfreundlichen Modellen im Arbeitsprozess gehalten werden.

Das Wissen der Medizin erweitert sich so schnell, dass es bereits heute für einen Menschen unmöglich ist, alle Publikationen seines Fachgebietes zu lesen. Schon bald werden KI-basierte Entscheidungsunterstützungssysteme mehr Wissen in sich vereinen, als ein menschlicher Facharzt. In der Folge werden Diagnosen und Therapievorschläge zunehmend von Maschinen erstellt werden, die zunächst von Ärzten nur noch überprüft und freigegeben werden, bevor dann die KI-Systeme vollautonom handeln werden. Ziel aktueller Entwicklungen ist die Verknüpfung von Sensoren und KI zu einem handlichen System für den Patientengebrauch, mit dem mehrere Krankheitsbilder unabhängig vom Arzt diagnostiziert werden können. Der Patient wird unabhängiger und Ärzte können sich auf anspruchsvollere Aufgaben konzentrieren. Radiologen, Pathologen und Kardiologen werden bei der Bildbefundung entlastet und später ganz ersetzt. Dadurch wird sich der Schwerpunkt dieser Tätigkeiten mehr auf den interventionellen Bereich verlagern.

\section{Pflegeberufe}

Berufe der Kranken-, Alten- und ambulanten Pflege gehen mit hohen physischen Anforderungen einher. Roboter entlasten die Menschen von diesen schweren körperlichen Tätigkeiten. Sie können Patienten heben und stützen und in Form von Exoskeletten den Patienten sogar körperliche Fähigkeiten zurückgeben, sodass sie weniger auf pflegerische Unterstützung angewiesen sind. Pflegeberufe haben dadurch mehr Zeit für die zwischenmenschlichen Aspekte ihrer Tätigkeit und Patienten werden länger zu Hause autonom leben können.

\section{Assistenzberufe und technische Berufe}

Mit der Unterstützung von Kommunikationstechnik, Sensoren und künstlicher Intelligenz können mehr ärztliche Tätigkeiten an die Assistenzberufe delegiert werden, was die Ärzte entlastet und diese Berufsgruppen aufwertet und attraktiver gestaltet.

Im Bereich der Zahntechnik wird sich das Berufsbild wandeln von den handwerklichen Fähigkeiten hin zu den IT-Fähigkeiten im Zusammenhang mit 3D-Druck. Es ist auch vorstellbar, dass zahntechnische Arbeiten vollständig von Zahnärzten mit der entsprechenden technischen Unterstützung durchgeführt werden können.

Der Beruf des Klinik-IT-Leiters wird sich weiterentwickeln hin zum Chief Digital Officer (CDO), der neben dem Betrieb der IT zuständig ist für die gesamte Digitale Transformationsstrategie des Krankenhauses, einschließlich vernetzter Medizintechnik und Social Media Management.

Neue Berufsbilder werden im Gesundheitswesen zum Einsatz kommen, wie beispielsweise Telemedizinische Versorgungsassistenten, Robomechatroniker und 3D-Ingenieure. 


\section{Berufe bei Krankenversicherungen}

Die Fähigkeit von Computern, Dokumente in natürlicher Sprache zu verstehen und zu verarbeiten, führt bereits heute dazu, dass Versicherungen Mitarbeiter durch Software ersetzen. Mittelfristig ist zu erwarten, dass alle einfachen Sachbearbeitertätigkeiten, einschließlich Call Centern, von Maschinen übernommen werden. Die entsprechenden Berufsgruppen werden sich auf höherwertige Aufgaben konzentrieren.

\section{Chancen und Risiken}

\section{Chancen}

E-Health-Technologien entlasten die Berufe im Gesundheitswesen von Routinetätigkeiten. Die gewonnene Zeit kann für anspruchsvollere Aufgaben, für die zwischenmenschlichen Aspekte der Gesundheitsversorgung oder für die schnellere und bessere Behandlung von mehr Patienten in Zeiten des demografischen Wandels und des relativen bzw. absoluten Mangels an Heilberuflern genutzt werden.

Diagnosen werden schneller und genauer, Therapien individueller. Die Technik kennt keine Müdigkeit und steht rund um die Uhr an allen Tagen zur Verfügung.

\section{Risiken}

Hochentwickelte Technik kann anfällig sein für Missbrauch. Daher müssen Qualität, Datenschutz, Datensicherheit und Schutz vor Manipulationen bei Entwicklung und Einsatz der Systeme eine hohe Priorität haben.

Die Nutzung der E-Health-Systeme sollte sich vorrangig an der Verbesserung des Patientenwohls orientieren und nicht allein von ökonomischen Aspekten bestimmt werden.

\section{Trends und Entwicklung}

Miniaturisierung und Preisverfall tragen dazu bei, dass der Patient eine zunehmend führende Rolle als Quelle und Verwalter seiner Gesundheitsdaten spielen wird. Durch künstliche Intelligenz wird er Unterstützung bei der Interpretation seines Gesundheitszustandes erhalten. Über Kommunikationssysteme wählt er seine Behandler unabhängig von der Entfernung aus. Somit wird der Patient zur zentralen Figur im Gesundheitswesen. Das Profil der Gesundheitsberufe wird sich wandeln in Richtung auf höherwertige Anforderungen, die noch nicht durch E-Health-Systeme erfüllt werden können. Ebenso werden neue Berufsbilder im Zuge der Digitalen Transformation entstehen.

Damit die Patienten von den Vorteilen der technischen Entwicklungen profitieren können, müssen die rechtlich-regulatorischen Rahmenbedingungen stimmen. Hier hat Deutschland dringenden Handlungsbedarf, um seinen mehrjährigen Rückstand auf seine europäischen Nachbarn, wie Schweiz, skandinavische Länder, Großbritannien und die Niederlande, aufzuholen. 
II Rahmenbedingungen

Um den Wandel in den Gesundheitsberufen erfolgreich bewältigen zu können, ist die Integration von E-Health in die Ausbildungscurricula der einzelnen Berufsbilder notwendig. 